\section{OP19 CONTENT ANALYSIS OF ADVANCE DIRECTIVES COMPLETED BY PATIENTS AS PART OF ADVANCE CARE PLANNING: INSIGHTS GAINED FROM THE ACTION TRIAL}

${ }^{1} \mathrm{M}$ Zwakman*, ${ }^{1} \mathrm{JJM}$ van Delden, ${ }^{2} \mathrm{G}$ Caswell, ${ }^{3} \mathrm{CA}$ Christensen, ${ }^{4} \mathrm{~L}$ Deliens, ${ }^{5} \mathrm{~F}$ Ingravallo, ${ }^{6} \mathrm{U}$ Jabbarian, ${ }^{7} \mathrm{AT}$ Johnsen, ${ }^{6} \mathrm{JJ}$ Korfage, ${ }^{8} \mathrm{~A}$ Mimic, ${ }^{9} \mathrm{NJ}$ Preston, ${ }^{1} \mathrm{MC}$ Kars. ${ }^{1}$ University Medical Center Utrecht, Utrecht, Netherlands; ${ }^{2}$ University of Nottingham, Nottingham, UK; ${ }^{3}$ University of Copenhagen, Copenhagen, Denmark; ${ }^{4}$ Vrije Universiteit Brussel, Brussel, Belgium; ${ }^{5}$ University of Bologna, Bologna, Italy; ${ }^{6}$ Erasmus University Medical Center, Rotterdam, Netherlands; 'Bispebjerg and Frederiksberg Hospital, Copenhagen, Denmark; ${ }^{8}$ University Clinic for Respiratory and Allergic Diseases Golnik, Golnik, Slovenia; ${ }^{9}$ Lancaster University, Lancaster, UK

\subsection{6/spcare-2019-ACPICONGRESSABS.19}

Background Writing an Advance Directive (AD) is often seen as a part of Advance Care Planning (ACP). ADs may include specific preferences regarding future care and treatment and information that provides a context for healthcare professionals and relatives in case they have to make decisions for the patient. The aim of this study was to get insight into the content of $\mathrm{ADs}$ as completed by patients with advanced cancer who participated in ACP conversations.

Methods A content analysis and descriptive statistics were conducted to describe the content of the completed My Preferences forms, an $\mathrm{AD}$ used in the intervention arm of the ACTION trial, testing the effectiveness of the ACTION Respecting Choices ACP intervention.

Results In total, 33\% of 439 patients who received the ACTION RC ACP intervention completed a My Preferences form. Document completion varied per country: 9.6\% (United Kingdom), 21\% (Denmark), 27.6\% (Belgium), $43.8 \%$ (the Netherlands), 61.3\% (Italy) and 64.3\% (Slovenia). Content analysis showed that 'maintaining normal life' and 'experiencing meaningful relationships' were important for patients to live well. Fears and worries mainly concerned disease progression, pain or becoming dependent. Patients hoped for prolongation of life and to be looked after by healthcare professionals. Most patients preferred to be resuscitated and $44 \%$ of the patients expressed maximizing comfort as their goal of future care. Most patients preferred 'home' as final place of care.

Conclusions My Preferences forms provide some insights into patients' perspectives and preferences. However, understanding the reasoning behind preferences requires conversations with patients.

\section{OP20 DOCTORS' PERSPECTIVES ON ADHERING TO ADVANCE CARE DIRECTIVES WHEN MAKING MEDICAL DECISIONS FOR PATIENTS WITH CHRONIC DISEASE:AN AUSTRALIAN INTERVIEW STUDY}

K Detering*, N Moore, T Low, L Nolte, M Sellars. Advance Care Planning Australia, Melbourne, Australia

\subsection{6/spcare-2019-ACPICONGRESSABS.20}

Background Advance care planning (ACP) assists people to identify their goals, values and treatment preferences for future care. Ideally documentation in an advance care directive (ACD) occurs. ACDs are used when treatment plans are developed for non-competent patients. Limited studies have explored perspectives of doctors regarding adherence to ACDs during medical decision-making for patients.
Aim To describe the perspectives and attitudes of doctors on adhering to ACDs.

Methods Doctors were recruited using convenience sampling. Face-to-face semi-structured interviews were conducted, audiotaped, transcribed and analysed using thematic analysis.

Interviews explored 3 case scenarios; doctors were asked to provide treatment plans. They were then given a values-based, followed by a treatment-based ACD. Prompting was used to understand how (if at all) the ACD influences treatment, and reasoning for decisions.

Results 21 doctors; median: 10 years experience; were included. Interviews lasted 10-60 minutes. Most doctors reported experience with ACP and ACDs. 3 major themes were identified: aligning with patient preferences (alleviating burden of decision-making, returning to baseline health, clarifying with others), questioning validity (distrusting patients' decision-making ability, navigating unrealistic goals, reaching ceiling of care) and navigating decisional conflict (prioritising best interest of the patient and overcoming family opposition).

Conclusion ACDs provide doctors with opportunities to align patient preferences with treatment; however, doctors sometimes question the validity of ACDs and experience decisional conflict when attempting to adhere to ACDs in practice, especially when family members oppose the plan. These results will assist development of clinical education programs, and resources supporting completion of more specific ACDs.

\section{OP21 PREVALENCE OF ADVANCE CARE PLANNING DOCUMENTATION AND SELF-REPORTED UPTAKE IN ONCOLOGY PATIENTS: FINDINGS FROM A NATIONAL AUSTRALIAN SURVEY}

${ }^{1}$ SM Blaschke, ${ }^{2} \mathrm{M}$ Sellars, ${ }^{2} \mathrm{~K}$ Detering, ${ }^{2} \mathrm{H}$ Kelly, ${ }^{2} \mathrm{~L}$ Nolte* ${ }^{*}$ Peter MacCallum Cancer Centre, Melbourne, Australia; ${ }^{2}$ Austin Hospital, Melbourne, Australia

\subsection{6/spcare-2019-ACPICONGRESSABS.21}

Background Absence of data on Advanced Care Directive (ACD) completion makes evaluation of Advance Care Planning (ACP) implementation challenging. This study reports the prevalence, accessibility and characteristics of ACDs for people with cancer aged 365 years from Australian hospital, primary care and aged care facilities.

Methods Prospective cross-jurisdictional study consisting of health record audit and self-report survey.

Result 51 sites and 2285 participants were included; 458 of whom had cancer. Of these, $27 \%$ had an ACD located in their record; $3 \%$ statutory stated preferences for care, $10 \%$ appointed substitute decision-maker, and 19\% were non-statutory document. Most (83\%) treatment plans were consistent with ACD preferences. More participants with cancer were receiving palliative care $(11 \%)$ compared to the overall sample (4\%). 97 participants with cancer completed the survey, 58\% reported they had completed ACP documentation; 30\% indicated trusting their children to make medical decisions; $26 \%$ trusted their partners, and 19\% their doctors.

Conclusion(s) This study is the first to examine ACP documentation prevalence in an Australian cancer population, at the point of care, and across jurisdictions. Whilst $27 \%$ of participants had an ACD located during the audit, more people self-reported having completed ACP documentation, 
which is encouraging as documentation available at point of care is likely to influence care. Only a low percentage of people were recorded as receiving palliative care. Recommendations to improve ACP uptake include: workforce education, care pathway inclusive of ACP and accessibility should be supported by national systems such as "My Health Record".

\section{OP22 CONSIDERATION AND IMPACT OF ADVANCE DIRECTIVES ON THERAPEUTIC DECISIONS IN TEN INTENSIVE CARE UNITS IN GERMAN SPEAKING SWITZERLAND}

${ }^{1} B$ Affolter*, ${ }^{2} \mathrm{~S}$ Schefold, ${ }^{2} S$ Eychmüller. ${ }^{1}$ Centre for Palliative Care, Berne, Switzerland; ${ }^{2}$ University Hospital of Berne, Berne, Switzerland

10.1136/spcare-2019-ACPICONGRESSABS.22

Background Since 2013 advance directives (AD) are binding in Switzerland. Regularly ICU doctors complain that they are not helpful for making therapeutic choices. But there is no data how often intensive care staff ask for advance directives at all and whether this represents the experience of the majority of intensive care doctors and nurses.

Methods We performed a survey in 10 Intensive Care Units (ICUs) from the German speaking part of Switzerland. The survey was sent either electronically or in paper format to 1041 professionals. Analysis was descriptive.

Results 355 (34\% response rate) professionals completed the questions concerning advance directives. Of the respondents $82 \%$ were nurses, $18 \%$ were ICU specialists or residents working in ICU. The majority of the staff asks always $(33 \%)$ or regularly $(50 \%)$ whether their patients have an AD. $6 \%$ of the doctors and $3 \%$ of the nurses say that an $\mathrm{AD}$ is always helpful for treatment decisions, while $29 \%$ of the doctors and $33 \%$ of the nurses judge it as usually helpful. $60 \%$ of doctors and $53 \%$ of nurses rated them as sometimes helpful for making therapeutic choices, while 4\% of doctors and $11 \%$ of nurses estimated ADs did not or hardly ever assisted them.

Conclusions In our survey, $83 \%$ of participants stated that they usually ask their patient or their relatives whether an $\mathrm{AD}$ exists. In contrast to the impressions from public media and personal conversations the majority of the respondents indicated that $\mathrm{ADs}$ are at least sometimes helpful for therapeutic decisions.

\section{OP23 PERCEPTIONS OF REASONS FOR DISCORDANCE BETWEEN CURRENT PREFERENCES AND EXISTING DOCUMENTATION: A QUALITATIVE STUDY}

S Hickman*, A Torke, A Myers, N Heim. Indiana University, Indianapolis, USA

\subsection{6/spcare-2019-ACPICONGRESSABS.23}

Background Nursing homes throughout the United States use the Physician Orders for Life-Sustaining Treatment (POLST) form to document resident treatment preferences as medical orders. Reasons for discordance between POLST orders and current preferences were explored in the context of a larger study of POLST discordance.

Methods Nursing facility residents $(n=25)$ and surrogate decision-makers $(n=25)$ were interviewed using Respecting
Choices Advanced Steps, a structured, advance care planning facilitation process, to elicit current values-based, informed preferences. Interviews were recorded, transcribed, and coded using qualitative descriptive methods.

Results Reasons for discordance between current preferences and POLST orders included changes in preferences related to new experiences, observations of other residents, new insights, a re-evaluation of goals and values, or a change in condition. Several participants indicated that discordance occurred because they learned new information or developed a better understanding about the treatment options during the interview. A few reported that the decisions recorded on POLST were influenced by others, though this was infrequent. Other participants were unable to explain the discordance because they did not recall the original conversation, or had no insight. Facility process issues including documentation errors and being asked to make decisions hastily during admission to the nursing facility also contributed to discordance.

Discussion Reasons for discordance are varied, but suggest a need for increased education, more frequent re-evaluation of treatment preferences, and improved practices in order to ensure nursing home documentation is concordant with the current, values-based informed preferences of residents.

\section{OP24 USAGE OF DO-NOT-ATTEMPT-TO RESUSCITATE-ORDERS IN A SWEDISH COMMUNITY HOSPITAL - PATIENT INVOLVEMENT, DOCUMENTATION AND COMPLIANCE}

1) Carlsson*, ${ }^{1} \mathrm{E}$ Bertilsson, ${ }^{2} \mathrm{~B}$ Semark, ${ }^{2} \mathrm{~K}$ Schildmeijer, ${ }^{2} \mathrm{~A}$ Bremer. ${ }^{1} \mathrm{~K} a$ lmar County Hospital, Kalmar, Sweden; ${ }^{2}$ Linnaeus University, Kalmar, Sweden

\subsection{6/spcare-2019-ACPICONGRESSABS.24}

Background The purpose of the study was to characterize patients dying in a community hospital with or without attempting cardiopulmonary resuscitation (CPR) and to describe patient involvement in, documentation of, and compliance with decisions on resuscitation (Do not attempt to resuscitate orders; DNAR).

Material and methods All patients who died in Kalmar County Hospital during January 1, 2016 until December 31, 2016 were included. All information from the patients' electronic chart was analyzed. Approved by the Regional Ethic review Board in Linköping, Sweden.

Results Of 660 patients (mean age $77.7 \pm 12.1$ years; range 21-101; median 79; 321 (48.6\%) female), a DNAR order had been documented in 563 patients (85.3\%). In 66 of 94 (70.2\%) patients without DNAR, CPR was unsuccessfully attempted. 28 of $94(29.8 \%)$ patients died without attempt at CPR and without a DNAR order in place. In 4 of 563 (0.7\%) patients CPR was attempted despite a DNAR order in place. In 416 patients $(73.9 \%)$ the DNAR order had not been discussed with neither patient nor family/friends. Moreover, in 84 cases $(14.9 \%)$ neither patient nor family/friends were even informed about the decision on code status.

Conclusions In general, a large percentage of patients in our study had a DNAR order in place (85.3\%). However, 28 patients $(4.2 \%)$ died without CPR attempt or DNAR order and DNAR orders had not been discussed with the patient/ surrogate in almost three fourths of the patients. Further work has to be done to implement ethical CPR guidelines to insure patient autonomy. 